Article

\title{
Acetylated Deoxynivalenol Generates Differences of Gene Expression that Discriminate Trichothecene Toxicity
}

\author{
Tadahiro Suzuki and Yumiko Iwahashi * \\ Applied Microbiology Division, National Food Research Institute, \\ National Agriculture and Food Research Organization (NARO), 2-1-12 Kannon-dai, \\ Tsukuba, Ibaraki 305-8642, Japan; suzut@affrc.go.jp \\ * Correspondence: yumiko@affrc.go.jp; Tel.: +81-29-838-8103; Fax: +81-29-838-7996 \\ Academic Editor: Sven Dänicke \\ Received: 11 December 2015; Accepted: 3 February 2016; Published: 6 February 2016
}

\begin{abstract}
Deoxynivalenol (DON), which is a toxic secondary metabolite generated by Fusarium species, is synthesized through two separate acetylation pathways. Both acetylation derivatives, 3-acetyl-DON (3ADON) and 15-acetyl-DON (15ADON), also contaminate grain and corn widely. These derivatives are deacetylated via a variety of processes after ingestion, so it has been suggested that they have the same toxicity as DON. However, in the intestinal entry region such as the duodenum, the derivatives might come into contact with intestinal epithelium cells because metabolism by microflora or import into the body has not progressed. Therefore, the differences of toxicity between DON and these derivatives need to be investigated. Here, we observed gene expression changes in the yeast $p d r 5 \Delta$ mutant strain under concentration-dependent mycotoxin exposure conditions. 15ADON exposure induced significant gene expression changes and DON exposure generally had a similar but smaller effect. However, the glucose transporter genes HXT2 and HXT4 showed converse trends. 3ADON also induced a different expression trend in these genes than DON and 15ADON. These differences in gene expression suggest that DON and its derivatives have different effects on cells.
\end{abstract}

Keywords: DON; acetylated derivative; toxicity; gene expression

\section{Introduction}

Deoxynivalenol (DON) is a secondary metabolite synthesized by Fusarium species, and is a mycotoxin that has various toxic effects. $\mathrm{DON}$, which is a type $\mathrm{B}$ trichothecene mycotoxin, is restricted in a number of countries, and regulatory limits have been established. The major activity of DON is binding to ribosome subunits, which affects cellular translation control. Because of this abnormal translation, cell proliferation is inhibited and disorder of physiological functions is induced. In the mammalian body, the region around the intestinal epithelia is very likely to be affected by DON. Cell lesions present as a toxicity phenotype through inflammation derived from enhanced inflammatory cytokines [1,2], and it is thought that DON induces abdominal pain or diarrhea. In yeast cells, which are used as a model in eukaryotic cell studies, some type B trichothecenes including DON affect the expression of ribosomal protein coding genes localized in both the cytosol and mitochondria [3]. Trichothecin, which belongs to the trichothecene mycotoxin family, has also been related to yeast mitochondrial gene expression changes [4]. However, DON exposure induces chlorosis in the leaves of Arabidopsis thaliana [5]. In this manner, DON shows various effects on different organisms. The DON synthesis pathway generates a number of derivatives that have this toxicity, though they differ in intensity. Acetylated DONs, such as 3-acetyl-DON (3ADON) and 15-acetyl-DON (15ADON) (Figure 1), are one type of derivative, and 
their environmental abundance ratios should not be ignored. Acetylated DONs are usually found at quite low levels, though high amounts of 3ADON compared with DON have occasionally been found in field experiments [6]. These acetylation types, 3A and 15A, are selectively decided in the DON synthesis pathway. Chemotypes, which are an expedient taxonomic designation, have been advocated for these acetylated products, and worldwide long-term experiments suggest that the composition of chemotypes changes repeatedly [7]. Therefore, it is necessary to better understand the characteristics of both DON and acetyl-DONs, and to consider their effects on organisms. In fact, one viewpoint is that the acetylation of DON is a transient detoxification mechanism for preventing autotoxicity. Indeed, 3-acetylation represents an effective detoxification, and previous experiments have indicated that 3ADON does not have any significant toxic phenotypes; however, 15ADON showed high toxicities to various cell lines [8-10]. Hence, except in fungal cells, it is thought that 15-acetylation enhances DON toxicity. In a previous study, we tested DON, 3ADON, 15ADON, nivalenol (NIV) and 4-acetyl-nivalenonl (4ANIV) using yeast cells, and investigated gene expression changes by DNA microarray (Figure 2) [3]. DON, 15ADON, and 4ANIV showed high toxicity, so acetylation was not linked to the degree of toxicity. Additionally, NIV, which shows high toxicity to several mammalian cell lines, did not present significant toxicity. Chlamydomonas reinhardtii, which is a green alga and has moderate sensitivity to NIV, was also used for a trichothecene exposure experiment [11]. However, 3ADON had no significant negative effects on any cell line.

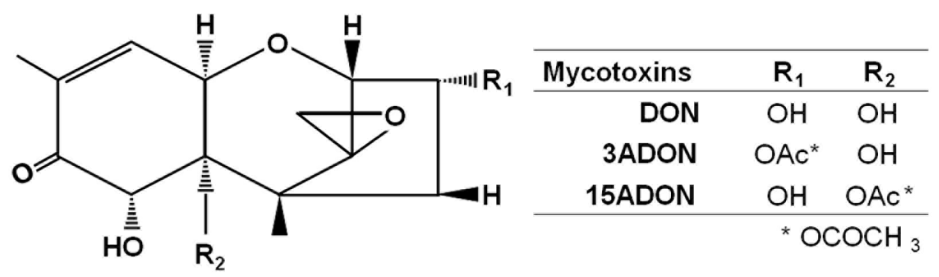

Figure 1. Deoxynivalenol and its derivatives.

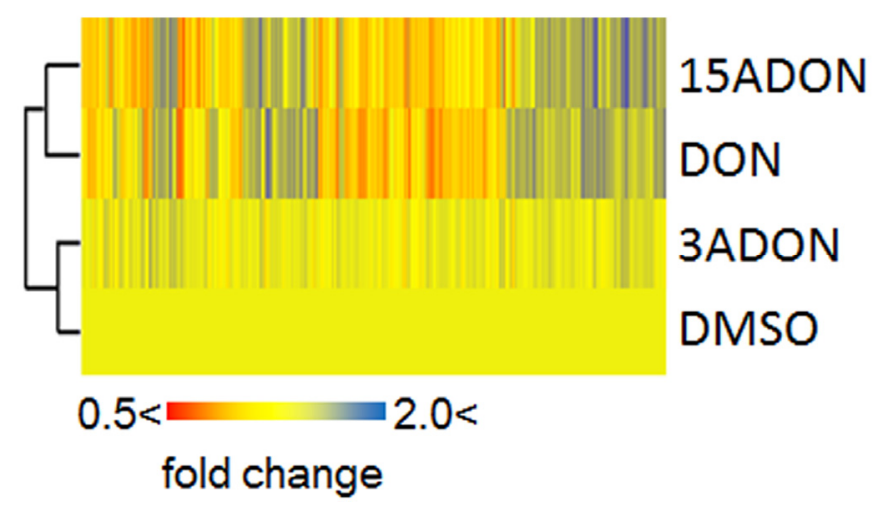

Figure 2. Comprehensive gene expression changes caused by mycotoxin exposure. DNA microarray data for the control sample (dimethyl sulfoxide; DMSO) and mycotoxin-exposed samples ( $25 \mathrm{mg} / \mathrm{L}$ (=25 ppm) deoxynivalenol; DON, 3-acetyl-DON; 3ADON, and 15ADON) were taken from a previous analysis (accession number GSE36954) [3]. These data were used to reconstruct a hierarchical map. Non-significant gene expression changes are indicated in yellow. Induced genes are represented in red; conversely, reduced genes are represented in blue.

Acetylated DON is imported from the digestive apparatus, and is then de-acetylated before it arrives at organs such as the liver. In such instances, it is thought that DON and its derivatives have a consistent toxicity mechanism against organisms. This suggests that a regulatory limit dependent on only the DON concentration is a problem because acetylated derivatives are ignored. Additionally, DON is de-epoxidized by intestinal microflora, and there is no convincing evidence that de-epoxy 
DON has toxic characteristics $[12,13]$. Therefore, the toxicity of these trichothecenes to an organism is considerably influenced by the organism's microflora. Because digestion by microflora and import to the organs have not progressed much, the region around the duodenal intestinal epithelium is thought to be susceptible to inflammation. At the same time, this means that DON derivatives retain their structure, which implies that derivatives induce toxicity through their own structures. If this is the case, it is necessary to identify specific derivative-induced toxic characteristics. In our previous research, we compared the comprehensive gene expression changes in yeast cells exposed to DON or its derivatives by DNA microarray analysis [3]. Yeasts do not have a deacetylation enzyme that reacts to DON derivatives; thus, the gene expression changes should represent derivative-specific toxic characteristics if the efficiency of intermembrane transfer is ignored. In that research, a mutant strain of pleiotropic drug resistance (PDR) transporter 5, which contributes substantially to type B trichothecene export, was used, and sodium dodecyl sulfate was added to increase the membrane permeability of mycotoxins. Even so, 3ADON exposure did not present a significant toxic phenotype or induce gene expression changes. Thus, it seems likely that structural changes derived from acetylation generate toxicity differences. The European Food Safety Authority (EFSA) reported that 15ADON represented up to $15 \%$ of DON contamination of agricultural products [14]. Additionally, 15ADON has shown high toxicity in various cell studies [3,5,11,12], so it should not be ignored. However, it is uncertain whether the differences in gene expression correspond to toxicity level changes because a number of genes, such as regulatory genes that encode transcription factors, often show temporary or rapid changes. A time-series experiment could solve this problem, but highly toxic agents prevent cell growth over time. Therefore, the gene expression changes will not provide clear information. Another way to obtain continuous gene expression data is with a concentration-dependent exposure experiment, which, through gene expression changes, could provide new insight into the differences in toxicity characteristics between DON and its derivatives. Here, we conducted a concentration-dependent exposure experiment using gene data that were extracted from a previous DNA microarray analysis [3]. DNA microarray analysis revealed significant expression changes derived from high-toxicity materials such as DON or 15ADON. However, it was insufficient to discriminate the differences in toxic characteristics, although the changes were useful for obtaining toxicity information about trichothecene mycotoxins. Therefore, we examined several new genes that showed different expression changes between trichothecenes, and identified concentration-dependent gene expression trends that could distinguish each mycotoxin.

\section{Results and Discussion}

\subsection{Influences of Pleiotropic Drug Resistance Transporter Regulation}

A pleiotropic drug resistance transporter gene-deletion mutant strain of yeast, $p d r 5 \Delta$, was used in this study. Pdr5p is a plasma membrane ATP-binding cassette (ABC) transporter of yeast, and functions as an efflux pump of various chemicals [15-18]. The strain pdr5 is a major DON-sensitive mutant, and a number of toxicity studies have been performed using this strain $[3,18,19]$. Additionally, it has been reported that the mutant of SNQ2, which is an ABC transporter (Table 1), has moderate sensitivity to mycotoxins [20]. Thus, both coding proteins, Pdr5p and Snq2p, are thought to be important for decreasing DON toxicity. The expression of these genes is regulated by the same transcription system as Pdr1p and Pdr3p (Figure 3a), so it is assumed that the expression of SNQ2 is up-regulated by the DON family in the $p d r 5 \Delta$ mutant. However, in a previous DNA microarray analysis, the expression of these genes did not show many significant changes. It is unclear whether changes of mycotoxin concentration affect the function of the PDR system in yeast cells. This may result in misinterpretation of the toxicity of trichothecene mycotoxins using the $p d r 5 \Delta$ mutant. We therefore observed the PDR1, $P D R 3$, and SNQ2 expression trends under various mycotoxin conditions. The expression of these genes was induced under DON and 15ADON conditions (Figure 3b). SNQ2 generally showed small expression changes (two-way factorial ANOVA; $p=0.30$ ), though its expression was suppressed under 
3ADON conditions (Student's $t$-test with DON or 15ADON, $p<0.01$ ) such that a precise view was not obtained. In contrast, relatively high induction of both PDR1 and PDR3 was observed under 15ADON conditions, while DON conditions showed lower induction compared with 15ADON. The expression of these transcription factors indicated that the toxicity of 15ADON was strong compared with that of DON. However, these expression change data did not reveal any hints to differentiate 15ADON from DON because the expression trends were similar. On the other hand, changing the mycotoxin concentrations did not lead to transient changes of gene expression patterns. Consequently, these data at least suggest that the yeast PDR system genes do not show transiently significant expression, which would prevent interpretation of the toxicity characteristics of these mycotoxins, and it is thought that experiments using $p d r 5 \Delta$ can reveal the toxicity of each mycotoxin adequately.

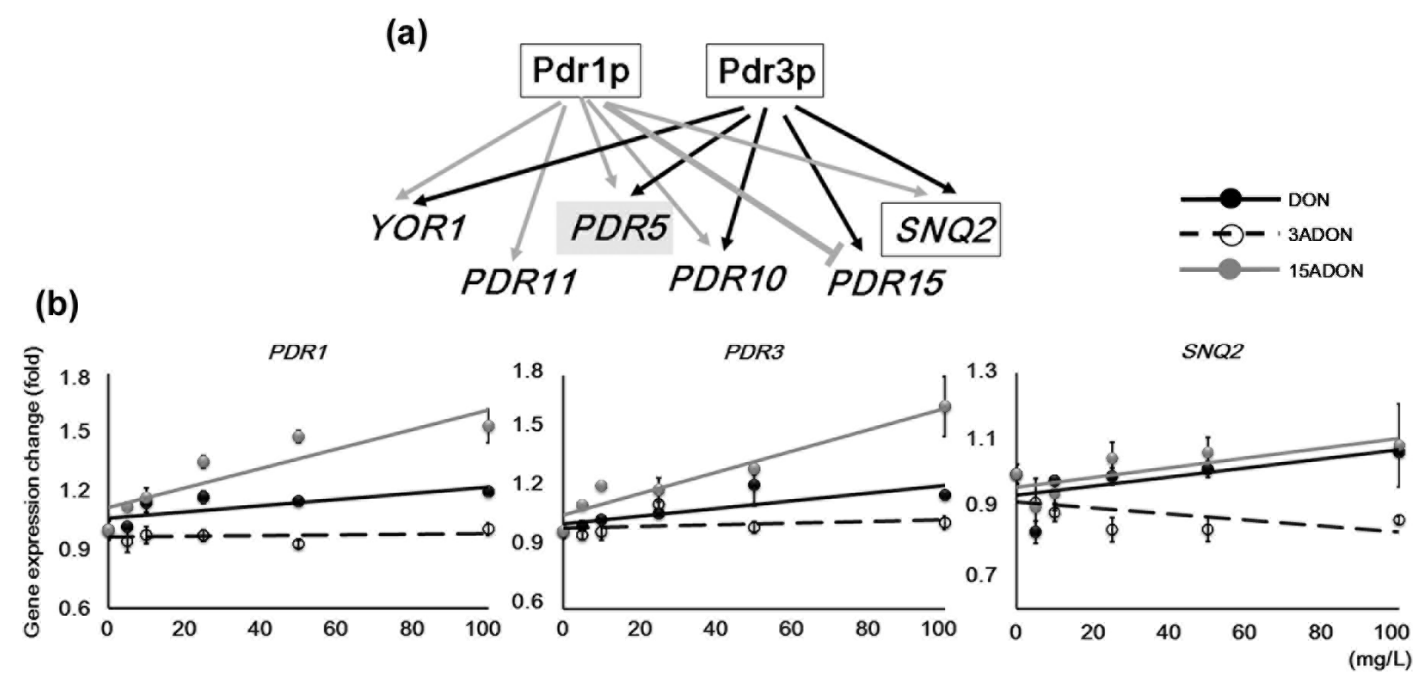

Figure 3. Expression changes of pleiotropic drug resistance (PDR) genes. (a) A simplified pleiotropic drug resistance regulation system, which was compiled by referring to a previous report [21]. Genes in boxes were examined in this study. Three capital letters with Italics indicates a gene; two small letters following a capital letter indicates a coding protein; (b) Concentration-dependent expression changes of PDR genes obtained by semi-quantitative polymerase chain reaction (PCR). ACT1 expression data in each sample were used for normalization. Approximate trend lines are shown. Bars = standard error; $n=3$. $p$-value of ANOVA < 0.01, except for SNQ2.

Table 1. Pleiotropic drug resistance-related genes observed in this study.

\begin{tabular}{cccc}
\hline $\begin{array}{c}\text { Systematic } \\
\text { Name }\end{array}$ & $\begin{array}{c}\text { Gene } \\
\text { Symbol }\end{array}$ & Localization & Description \\
\hline YGL013C & PDR1 & cytoplasm, nucleus & Regulates pleiotropic drug resistance \\
YBL005W & PDR3 & cytoplasm, nucleus & $\begin{array}{c}\text { Transcriptional activator of the pleiotropic drug resistance network } \\
\text { plasma membrane ATP binding cassette (ABC) transporter }\end{array}$ \\
YDR011W & SNQ2 & plasma membrane & Plasma mempons \\
\hline
\end{tabular}

\subsection{Stress-Responsive Redox Genes}

Since suitable PDR genes for discriminating DON and acetylated DON were not found, we focused on other stress response mechanisms. Yeast cells form an iron-sulfur (Fe-S) cluster that functions as an adjustor of redox reactions to prevent DNA damage caused by reactive oxygen species (ROS). To maintain this mechanism, cells import $\mathrm{Fe}^{2+}$, which is reduced from $\mathrm{Fe}^{3+}$, through cellular membrane transporters. Yeast has FRE genes that encode ferric/cupric reductases that localize to the plasma membrane, and these genes are roughly categorized into three families [22] (Table 2, Figure 4a). The enzymatic activities of both Fre1p and Fre2p cover almost all (>90\%) iron ion reduction activity at the plasma membrane [23]. Mammalian cells such as intestinal epithelium cells have a similar 
function; enteral $\mathrm{Fe}^{3+}$ is reduced to $\mathrm{Fe}^{2+}$, which is preserved as ferritin or used to regulate intravital oxygenic products. However, Fre1p and Fre2p are similar to gp91, a large subunit of cytochrome b-245, which is a component of human NADPH oxidase [23,24]. This human component synthesizes superoxide through the addition of an electron at the membrane surface, and this superoxide kills harmful extracellular microorganisms. A lack of gp91 causes depression of bactericidal activity, and induces X-linked chronic granulomatous disease. Conversely, excess gp91 activity causes ROS stress to its own cells. Thus, the monitoring of gp91 can help us understand changes in cellular oxidative stress. If Fre1p and Fre2p have the same function, FRE genes have the potential to be markers for the stress response at the cellular membrane surface of yeast. Therefore, we observed the expression of FRE1 and FRE2 (Figure 4b). Three additional genes, FRE7, FRE3, and FRE8, were also tested under the same conditions. FRE7, which is a FRE1 family member, did not show many significant changes, though DON exposure decreased its expression. For FRE1, more than $25 \mathrm{mg} / \mathrm{L}$ of both DON and 15ADON caused a significant reduction of expression. The expression of FRE2 and FRE3 (family I) showed significant concentration-dependent induction under 15ADON conditions, while DON exposure resulted in the same trend of a small expression increase. Although it had smaller expression changes, FRE8, which belongs to a different group from the above families, also showed a similar expression pattern to FRE2 and FRE3. Taken together, both DON and 15ADON reduced FRE1 expression, and induced FRE2 and FRE3. Moreover, for FRE2 and FRE3, 15ADON-induced gene expression changes were higher than those of DON. These results indicated contrasting expression between FRE1 and FRE2, and suggested that their roles or functions are different. This study might be the first report of contrasting gene expression patterns for FRE1 and FRE2, although it is known that they are induced by iron deficiency. Nonetheless, the FRE1 and FRE2 genes were not suitable for discriminating the toxicity characteristics of DON and 15ADON because the expression patterns of both were partially similar, while the expression trends of FRE3 corresponded to those of FRE2. As for FRE7, the expression trends differed under DON and 15ADON exposure. However, it would be difficult to discriminate the influence of each because the 15ADON-derived gene expression changes were not stable. Additionally, a lack of FRE7 has no specific effect [25], so Fre7p is thought to be an accessory enzyme. Overall, trichothecene exposure caused FRE gene expression changes, although this does not necessarily mean they can be used as markers for evaluating trichothecene toxicity. On the surface, the role of Fre seems contrary to that of gp91. However, both mechanisms contribute to stress reduction through redox reactions; thus, redox mechanisms are thought to be important for maintaining cellular components and processes across species.

Table 2. FRE family genes observed in this study.

\begin{tabular}{ccccccc}
\hline $\begin{array}{c}\text { Systematic } \\
\text { Name }\end{array}$ & $\begin{array}{c}\text { Gene } \\
\text { Symbol }\end{array}$ & Group * & \multicolumn{2}{c}{ Induction with } & Localization & Description \\
\cline { 4 - 5 } & & Low Level Iron & Low Level Copper & \\
\hline YLR214W & FRE1 & II & + & ++ & + & Ferric reductase and \\
YKL220C & FRE2 & I & ++ & + & plasma & $\begin{array}{c}\text { Ferric reductase } \\
\text { membrane }\end{array}$ \\
YOR381W & FRE3 & I & ++ & Ferric reductase \\
YOL152W & FRE7 & II & - & ++ & & $\begin{array}{c}\text { Ferric reductase and } \\
\text { cupric reductase }\end{array}$ \\
YLR047C & FRE8 & III & - & - & $\begin{array}{c}\text { Similarity to ferric/ } \\
\text { cupric reductases }\end{array}$ \\
\hline
\end{tabular}

* Group numbers were established in experiments [22]. +, ++ and - indicates the gene induction levels.

+ : induction; ++: high level induction; -: not induced or not confirmed. 


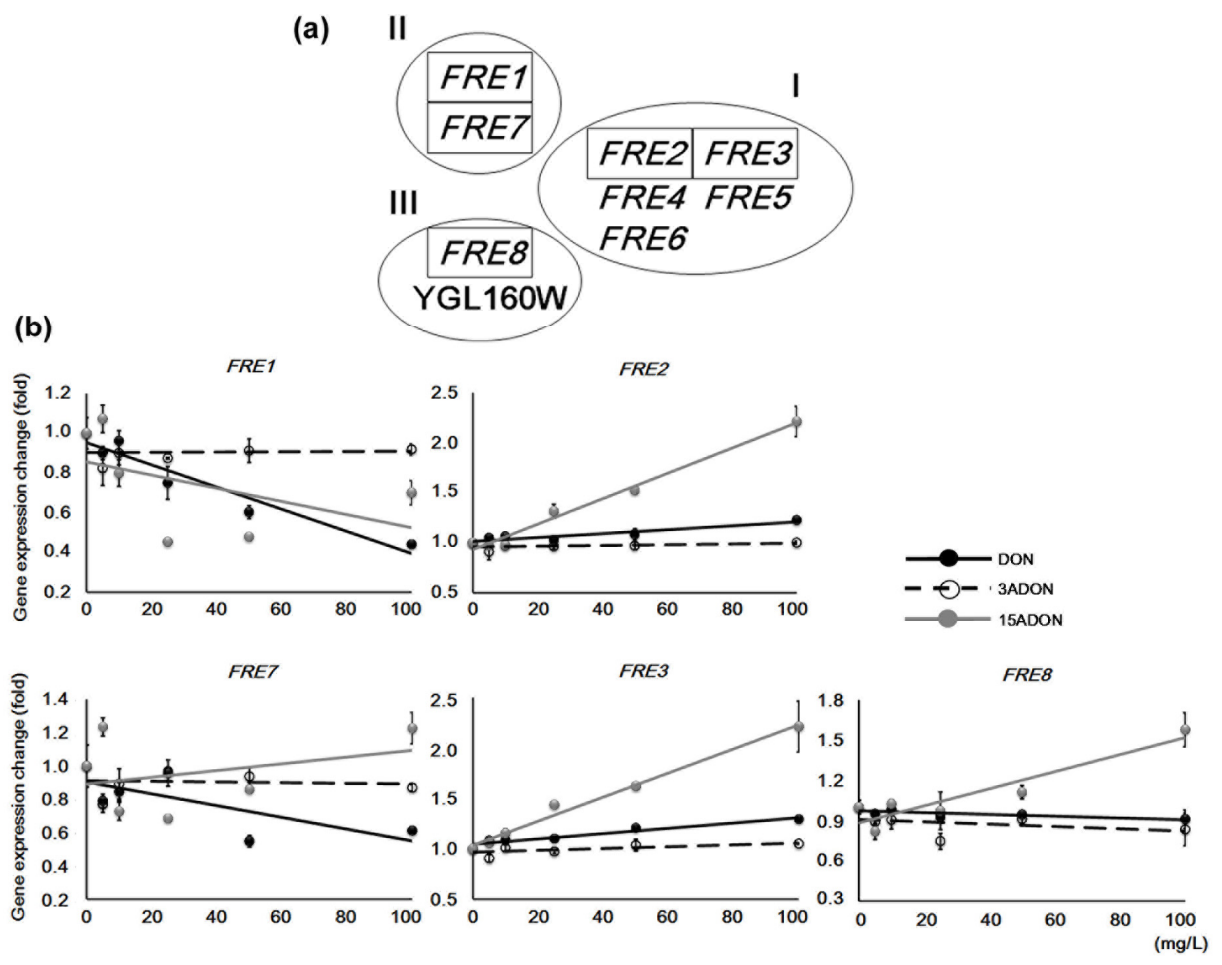

Figure 4. Expression changes of ferric reductase ( $F R E)$ genes. (a) Experimentally categorized families of FRE genes. Genes in boxes were examined in this study; (b) Concentration-dependent expression changes of FRE genes were identified by semi-quantitative PCR. ACT1 expression data in each sample were used for normalization. Approximate trend lines are shown. Bars = standard error; $n=3$. $p$-value of ANOVA $<0.01$.

\subsection{Genes Specifically Responsive to $15 A D O N$}

In investigating gene expression trends, we found various types of concentration-dependent changes. For example, the expression of several genes was reduced under higher concentrations but was induced under low concentration conditions. Such inconsistent expression changes can mislead gene expression analysis. To make interpretation easier, we next examined several genes that showed $15 \mathrm{ADON}$-specific expression changes (Table 3, Figure 5).
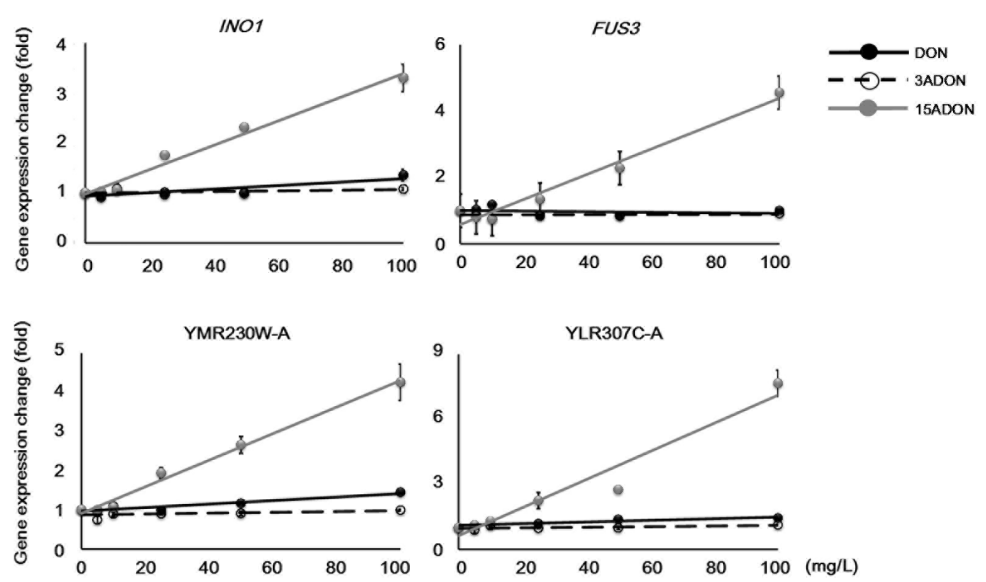

Figure 5. 15ADON-specific gene expression changes. Concentration-dependent gene expression changes were identified by semi-quantitative PCR. ACT1 expression data in each sample were used for normalization. Approximate trend lines are shown. Bars $=$ standard error; $n=3$. $p$-value of ANOVA $<0.01$. 
Table 3. Uncategorized genes showing 15ADON-specific expression changes.

\begin{tabular}{cccc}
\hline $\begin{array}{c}\text { Systematic } \\
\text { Name }\end{array}$ & $\begin{array}{c}\text { Gene } \\
\text { Symbol }\end{array}$ & Localization & Description \\
\hline YBL016W & FUS3 & cytoplasm, nucleus & Mitogen-activated serine/threonine protein kinase \\
YJL153C & INO1 & cytoplasm & Inositol-3-phosphate synthase \\
YLR307C-A & - & unknown & Uncharacterized \\
YMR230W-A & - & unknown & Uncharacterized \\
\hline
\end{tabular}

INO1 encodes an inositol-3-phosphate synthase involved in the synthesis of inositol phosphates (IPs), which are utilized in the sphingolipid metabolic pathway for maintaining various cellular processes. In the sphingolipid metabolic pathway, IPs are used as not only cellular components but also cell proliferation signals, and normal IP regulation is essential to maintain normal cellular processes. In a previous toxicity evaluation study of aflatoxin $\mathrm{B}_{1}\left(\mathrm{AFB}_{1}\right)$ using the yeast mutant strain of PTC1, which encodes an inhibitory regulation protein of the mitogen-activated protein kinase (MAPK) pathway, we observed significant up-regulation of INO1 [26]. Furthermore, in that study, the down-regulation of sphingolipid metabolism pathway genes was examined. In addition, it has been reported that fumonisin $\mathrm{B}_{1}\left(\mathrm{FB}_{1}\right)$, which is a mycotoxin synthesized by Fusarium sp., also inhibits ceramide synthase located in the sphingolipid metabolism pathway [27]. Because 15ADON induced INO1 in a concentration-dependent manner, it may affect the sphingolipid metabolism pathway such as $\mathrm{AFB}_{1}$ or $\mathrm{FB}_{1}$. FUS3 also encodes a serine/threonine-specific kinase of the MAPK pathway, which is activated as a stress response. Additionally, activation of Fus3 induces $\mathrm{G}_{1}$ arrest of the cell cycle [28]. In this study, FUS3 was induced by 15ADON exposure, suggesting cell cycle arrest. Given that DON-derived FUS3 induction was quite low, FUS3 might be a suitable marker for discriminating DON and 15ADON. The uncharacterized genes $Y L R 307 C-A$ and $Y M R 230 \mathrm{~W}-A$ showed similar results to FUS3. These genes do not have any known characteristics such as signal peptides, motifs, or transmembrane regions. However, they showed consistent expression trends, suggesting that they are influenced by some sort of functional gene damaged by mycotoxin exposure. Because FUS3 and YLR307C-A showed small expression changes under DON conditions, these genes especially could be superior candidates as markers for $15 \mathrm{ADON}$ toxicity.

\subsection{Glucose Transporter Genes Differentiate DON and Its Derivatives}

In yeast cell studies, $15 \mathrm{ADON}$ causes a large number of gene expression changes compared with other type B trichothecenes, and the change levels are also significant [3]. DON induced relatively small expression changes compared with $15 \mathrm{ADON}$ despite showing similar expression trends, such that DON-specific expression changes were not easily identified. However, some DON-specific gene expression changes were observed. In this study, we focused on the hexose transporter genes, HXT2 and HXT4, which localize to the plasma membrane such as FRE genes (Table 4, Figure 6a). The regulatory mechanisms of HXT1, HXT2, and HXT4 have been well investigated, and they are generally induced by changes of glucose level [29-31]. Glucose is transported from the extracellular space, or synthesized by gluconeogenesis, and then converted to inositol. Because of this glucose usage circulation, HXT genes were examined (Figure 6b). HXT1 and HXT2 were induced by DON exposure, whereas the other mycotoxins did not show significant gene expression induction. In addition, HXT2 and HXT4 showed a repression trend under 15ADON exposure while the opposite trend was observed under DON exposure. In this way, the trends under DON and 15ADON were clearly different, although under $100 \mathrm{mg} / \mathrm{L}$ conditions, HXT1 did not show any significant difference. Taken together, both HXT2 and HXT4 might be suitable genes for discriminating DON- and 15ADON-specific toxicity characteristics. Additionally, 3ADON exposure caused HXT2 repression and HXT4 induction, although the changes were small. These expression patterns differed from those of DON and 15ADON, so HXT2 and HXT4 could also be valuable genes for discriminating 3ADON-specific toxicity. The HXT genes have different regulation mechanisms; HXT1 encodes a low affinity glucose transporter that is induced 
by $>1 \%$ glucose conditions, while the rest are high affinity glucose transporters that are induced by $<0.2 \%$ glucose condition [31]. Additionally, our results suggested novel characteristics for these genes.

(a)

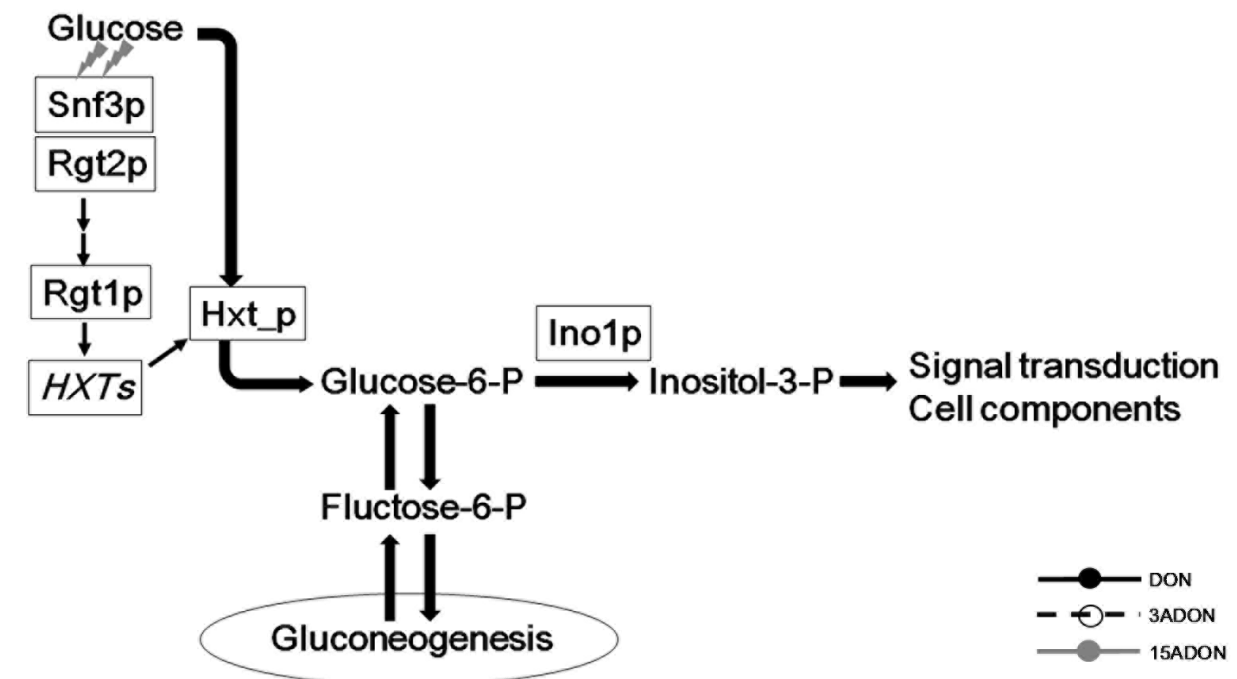

(b)
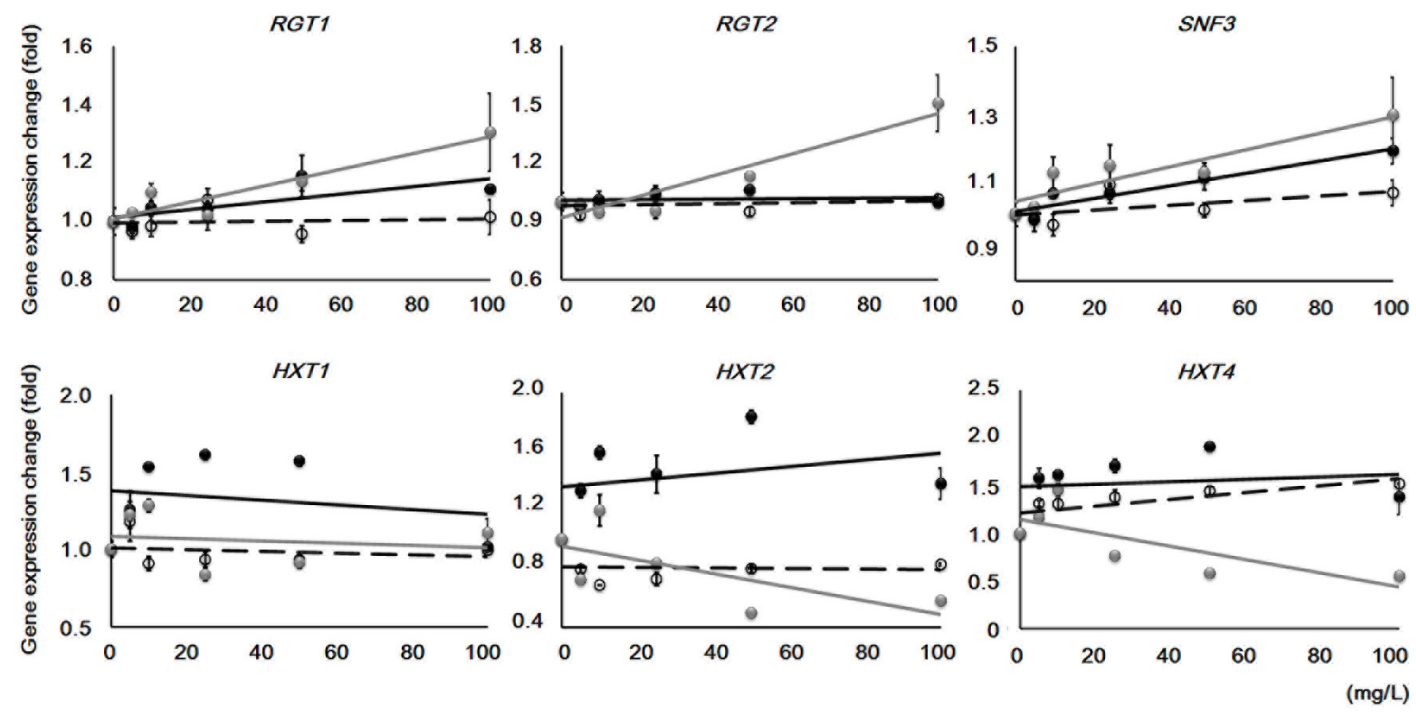

Figure 6. Expression changes in glucose import and utilization genes. (a) The transporter system and gluconeogenesis provide glucose, which is converted to inositol. Three capital letters with Italics indicate a gene; two small letters following a capital letter indicate a coding protein; (b) Concentration-dependent expression changes in glucose transporter and sensor or regulator genes were identified by semi-quantitative PCR. ACT1 expression data in each sample were used for normalization. Approximate trend lines are shown. Bars $=$ standard error; $n=3$. $p$-values of ANOVA: RGT1, $p<0.03$; other genes, $p<0.01$.

Table 4. Glucose-sensing and importer genes observed in this study.

\begin{tabular}{cccc}
\hline Systematic Name & Gene Symbol & Localization & Description \\
\hline YKL038W & RGT1 & nucleus & Glucose-responsive transcription factor \\
YDL138W & RGT2 & plasma membrane & Plasma membrane glucose sensor \\
YDL194W & SNF3 & plasma membrane & Plasma membrane low glucose sensor \\
YHR094C & HXT1 & plasma membrane & Low-affinity glucose transporter \\
YMR011W & HXT2 & plasma membrane & High-affinity glucose transporter \\
YHR092C & HXT4 & plasma membrane & High-affinity glucose transporter \\
\hline
\end{tabular}


The gene expression of $R G T 1, R G T 2$, and SNF3, which encode a component of a transcriptional regulator complex and glucose sensors, showed similar patterns to other transcriptional regulators such as PDR1 and PDR3. The inhibitory regulation of RGT1 on HXT genes is abolished by glucose sensing. However, the cells were cultured in YPD with each mycotoxin for only $2 \mathrm{~h}$, so there was a low possibility for glucose deficiency in the culture media. Because no glucose was supplied in the test conditions, the glucose transporter genes might have been regulated by a different trigger to glucose. Differences in gene expression trends between the three mycotoxins that were not linked to these regulatory genes directly suggested abnormal regulation mechanisms. Essentially, 15ADON exposure leads to higher toxicity than that of DON in yeast cell studies, and both DON and 15ADON induce similar trends of gene expression changes. However, for the HXT genes in this research, 15ADON showed negative correlations and DON induced these genes under all conditions, which was unusual. Taken together, this suggests that DON and $15 \mathrm{ADON}$ do not have completely corresponding toxicity.

\section{Conclusions}

As of 2010, the provisional maximum tolerable daily intake (PMTDI) established for DON was extended to acetyl-DON by the Joint FAO/WHO Expert Committee on Food Additives (JECFA) [32]. This is because acetyl-DON is metabolized in the body and the de-acetylation product is synthesized such that acetyl-DON is thought to potentially have the same risk as DON. However, the intestinal surface around the duodenum is at risk of being damaged by acetylated products directly because the de-acetylation reaction has not progressed or started yet. Therefore, understanding the differences in toxicity between DON and its acetylated products is important for the development of toxicity evaluation. This study suggests that the toxic effects of DON partially differ from those of 15ADON; thus, further investigation of the toxicity of such modified products is needed.

\section{Experimental Section}

\subsection{Chemicals}

Deoxynivalenol (DON), 3-acetyldeoxynivalenol (3ADON), and 15-acetyldeoxynivalenol (15ADON) powders (Wako, Osaka, Japan) were each dissolved in dimethyl sulfoxide (DMSO). Stock solutions were added into each medium to prepare test conditions.

\subsection{Cell Strain and Growth Conditions}

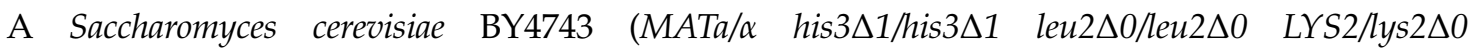
met15 $\Delta 0 / M E T 15 u r a 3 \Delta 0 /$ ura $3 \Delta 0$ ) derived PDR5 deletion mutant ( $p d r 5 \Delta$; Thermo Fisher Scientific, Waltham, MA, USA) with the loss of multidrug resistance transporter function was used in this study. Glycerol stocks, which were stored at $-80{ }^{\circ} \mathrm{C}$, of yeast cells were transferred with an inoculating needle into YPD media (1\% yeast extract, $2 \%$ peptone, and $2 \%$ glucose) into three conical flasks and incubated on a rotary shaker (TOKYO RIKAKIKAI, Tokyo, Japan) at $150 \mathrm{rpm}$ and $25^{\circ} \mathrm{C}$ for at least 2 days. The pre-culture solutions were measured with a spectrophotometer at $650 \mathrm{~nm}$. These solutions were transferred to fresh YPD to dilute the cell concentration, and were incubated on a rotary shaker at $150 \mathrm{rpm}$ and $25^{\circ} \mathrm{C}$ for several hours until the absorbance at $650 \mathrm{~nm}\left(\mathrm{~A}_{650}\right)=0.8-1.0$. Six milliliter aliquots of the prepared culture solutions were dispensed into test tubes, and a final concentration of $0.01 \%$ sodium dodecyl sulfate was added to enhance the cell permeability of the mycotoxins. The mycotoxins were added into the test tubes at final concentrations of 5, 10, 25, 50, and $100 \mathrm{mg} / \mathrm{L}$. DMSO, which was used to dissolve the mycotoxins, was added to a non-treatment control sample $(0 \mathrm{mg} / \mathrm{L})$. DMSO was also added to other samples so that the final concentration of each additive was $0.5 \%$. The prepared test tube samples were immediately incubated on a reciprocal shaker (TAITEC, Saitama, Japan) at 150 strokes/min and $25{ }^{\circ} \mathrm{C}$ for $2 \mathrm{~h}$. The culture media were transferred into $15 \mathrm{~mL}$ corning tubes, and cell pellets were obtained by centrifugation at $3000 \mathrm{rpm}$ for $7 \mathrm{~min}$ at $4{ }^{\circ} \mathrm{C}$. The pellets were immediately frozen with liquid nitrogen, and stored at $-80^{\circ} \mathrm{C}$. 


\subsection{RNA and cDNA Preparation, Semi-Quantitative PCR}

Total RNA was extracted from the cell pellets using a commercial kit (FastRNA Pro Red kit, MP Biomedicals, Irvine, CA, USA), following the manufacturer's instructions. To avoid genomic DNA contamination, the samples were treated with deoxyribonuclease (DNase I, Takara, Shiga, Japan) before reverse transcription. First-strand cloned DNA (cDNA) was synthesized with reverse transcriptase (Prime script II 1st strand cDNA synthesis kit, Takara, Japan) from the total RNA. Residual RNAs in the cDNA products were digested with ribonuclease A (RNaseA, Thermo Fisher Scientific, Waltham, MA, USA). Each cDNA product was diluted with two volumes of Tris-EDTA ( $\mathrm{pH}$ 8.0), and the cDNA solution was used as a template. Sequence information for the target genes was obtained from the Saccharomyces Genome Database (SGD) [33], and each primer set that corresponded to the position around the $3^{\prime}$-end sequence was manually designed (Table 5). The actin protein coding gene ACT1 was used as an internal control. An ACT1 primer set with the same sequence as in a previous report [34] was prepared. The other primer sets were designed by Primer3 [35]. To obtain a standard curve for each target gene, non-treatment control (NTC) cDNA was prepared and PCR was conducted to construct the standard curves. In this study, $20 \mu \mathrm{L}$ reaction solutions $(1 \mu \mathrm{L}$ of each $10 \mu \mathrm{M}$ primer, $2 \mu \mathrm{L}$ of $10 \times$ buffer, $1.6 \mu \mathrm{L}$ of $2.5 \mathrm{mM}$ dNTPs, $1 \mu \mathrm{L}$ of DNA template, $15.2 \mu \mathrm{L}$ of distilled water, and $0.2 \mu \mathrm{L}$ of Taq polymerase (Gene Taq, Nippon Gene, Tokyo, Japan) were prepared and subjected to $95^{\circ} \mathrm{C}$ for $5 \mathrm{~min}$, followed by 35 cycles of $95^{\circ} \mathrm{C}$ for $30 \mathrm{~s}, 55^{\circ} \mathrm{C}$ for $30 \mathrm{~s}$, and $72{ }^{\circ} \mathrm{C}$ for $60 \mathrm{~s}$ in a thermal cycler (C1000, Bio-Rad, Hercules, CA, USA). For each primer set, a template dilution series was prepared from the PCR products and $1 \mu \mathrm{L}$ of each sample was dispensed into a 96-well PCR plate. The same volume of mycotoxin-treated sample templates were also dispensed into the plate. A total of $19 \mu \mathrm{L}$ of reaction mix $(0.4 \mu \mathrm{L}$ of each $10 \mu \mathrm{M}$ primer, $8.2 \mu \mathrm{L}$ of distilled water, and $10 \mu \mathrm{L}$ of $2 \times$ Master mix (KAPA SYBR FAST qPCR kit, Kapa Biosystems, MA, USA)) was added to each well. The sample plate was subjected to $95^{\circ} \mathrm{C}$ for $20 \mathrm{~s}$, followed by 40 cycles of $95^{\circ} \mathrm{C}$ for $3 \mathrm{~s}$, and $60^{\circ} \mathrm{C}$ for $20 \mathrm{~s}$, in a thermal cycler (MX3000P, Agilent Technologies, Santa Clara, CA, USA). The amplified ACT1 product was used as an internal control, and triplicates were averaged. Part of a DNA microarray dataset (accession no. GSE36954) recorded in the Gene Expression Omnibus Database (GEO) was also used [36]. Two-way factorial ANOVA was conducted for each gene expression data series, and, under some concentration conditions, two facultative mycotoxin-exposed samples were compared by Student's $t$-test analysis.

Table 5. Primer sets used in this study.

\begin{tabular}{|c|c|c|}
\hline \multirow{2}{*}{ Name } & Direction & \multirow{2}{*}{ Forward or Reverse } \\
\hline & $5^{\prime}$ & \\
\hline \multirow{2}{*}{ ACT1 } & ATTGCCGAAAGAATGCAAAAGG & $\mathrm{F}$ \\
\hline & CGCACAAAAGCAGAGATTAGAAACA & $\mathrm{R}$ \\
\hline \multirow{2}{*}{ FRE1 } & GCTCGGAAATAAAACTCTCAGAA & $\mathrm{F}$ \\
\hline & ATTATTAACAAGGGGCCTTACCA & $\mathrm{R}$ \\
\hline \multirow{2}{*}{ FRE2 } & CGACCAAATGTTAAGGAACTTCTAC & $\mathrm{F}$ \\
\hline & AAATGATCACCAGCATTGATACTCT & $\mathrm{R}$ \\
\hline \multirow{2}{*}{ FRE3 } & TAAATGGATCGTTAGCTGTGGTT & $\mathrm{F}$ \\
\hline & TTGTGATAGGTAAAATAGTGAGGAAA & $\mathrm{R}$ \\
\hline \multirow{2}{*}{ FRE7 } & GCTTCATTTGTTCAGGTTCTGAC & $\mathrm{F}$ \\
\hline & TGCTTGAATGATATTTCACATGG & $\mathrm{R}$ \\
\hline \multirow{2}{*}{ FRE8 } & ACGGATTCTATTCAATGTTGCAG & $\mathrm{F}$ \\
\hline & TCAAATACGTGAATTTTCCAAGC & $\mathrm{R}$ \\
\hline \multirow{2}{*}{ FUS3 } & ATCCGTATTTGCAAACATACCAC & $\mathrm{F}$ \\
\hline & TGTATACATTGTTCTTCGGGTTGATA & $\mathrm{R}$ \\
\hline \multirow{2}{*}{ HXT1 } & CGTCTTTTTCTTCGTTCCAGA & $\mathrm{F}$ \\
\hline & GAGCTTGTTTAGTTTATTTCCTGCT & $\mathrm{R}$ \\
\hline \multirow{2}{*}{ HXT2 } & GGGTATGTCTTCATGGGCTGT & $\mathrm{F}$ \\
\hline & TATAATCTCTTATTCCTCGGAAACTC & $\mathrm{R}$ \\
\hline
\end{tabular}


Table 5. Cont

\begin{tabular}{|c|c|c|}
\hline \multirow{2}{*}{ Name } & Direction & \multirow{2}{*}{ Forward or Reverse } \\
\hline & $5^{\prime}$ & \\
\hline \multirow{2}{*}{ HXT4 } & TTCTTCGTTCCAGAAACTAAAGG & $\mathrm{F}$ \\
\hline & CCGAACATCTTCTTGTAAAATGG & $\mathrm{R}$ \\
\hline \multirow[b]{2}{*}{ INO1 } & AAGATGCTGGCAAATTCGAG & $\mathrm{F}$ \\
\hline & TGAGATTACAACAATCTCTCTTCG & $\mathrm{R}$ \\
\hline \multirow{2}{*}{ PDR1 } & ACAATATTAACAACAACAACAGTAACAA & $\mathrm{F}$ \\
\hline & GGAAGGAAGTTTTTGAGAACTTTTA & $\mathrm{R}$ \\
\hline \multirow[b]{2}{*}{ PDR3 } & CAACAGACAAAAAGACAACATTCTG & $\mathrm{F}$ \\
\hline & CCATTTACTATGGTTATGCTCTGCT & $\mathrm{R}$ \\
\hline \multirow{2}{*}{ RGT1 } & CCTCCGCGAGTCATCAGT & $\mathrm{F}$ \\
\hline & ACCTGTCAATACCAGCCTAACTC & $\mathrm{R}$ \\
\hline \multirow[b]{2}{*}{ RGT2 } & CAATAACAACACTGAACGAAATGG & $\mathrm{F}$ \\
\hline & GGGGAAGTGTATTGGCTGTG & $\mathrm{R}$ \\
\hline \multirow{2}{*}{ SNF3 } & GAACGAATGGCGCAGTTT & $\mathrm{F}$ \\
\hline & CAAATCATTATTTTCATTTACAGGTTG & $\mathrm{R}$ \\
\hline \multirow{2}{*}{ SNQ2 } & GCTACTTGTGGAGAAATTTTGGA & $\mathrm{F}$ \\
\hline & GCAGATGAATGCACAAAATGTTA & $\mathrm{R}$ \\
\hline \multirow{2}{*}{ YLR307C-A } & GGGATGCTAGTACTACTCAATGTCG & $\mathrm{F}$ \\
\hline & TTAATTCAAATTATACTTTTACGTGCTC & $\mathrm{R}$ \\
\hline \multirow{2}{*}{ YMR230W-A } & GGATGTGTTACGATGCAGACA & $\mathrm{F}$ \\
\hline & ATATGGCGCGTTCTTGAAGG & $\mathrm{R}$ \\
\hline
\end{tabular}

Acknowledgments: Part of this work was supported by the Japan Society for the Promotion of Science (JSPS) KAKENHI Grant Number 25871096.

Author Contributions: Tadahiro Suzuki conceived, designed and performed the experiments, and wrote the manuscript. Yumiko Iwahashi supervised the experiments and revised the manuscript.

Conflicts of Interest: The authors declare no conflict of interest.

\section{Nomenclature}

Three capital letters with Italics; gene

Two lowercase letters following a capital letter + p; coding protein

Three small letters with Italics $+\Delta$; mutant strain

ANOVA; analysis of variance

\section{References}

1. Pestka, J.J.; Zhou, H.R.; Moon, Y.; Chung, Y.J. Cellular and molecular mechanisms for immune modulation by deoxynivalenol and other trichothecenes: unraveling a paradox. Toxicol. Lett. 2004, 153, 61-73. [PubMed]

2. Pestka, J.J.; Uzarski, R.L.; Islam, Z. Induction of apoptosis and cytokine production in the Jurkathuman T cells by deoxynivalenol: Role of mitogen-activated protein kinases and comparison to other 8-ketotrichothecenes. Toxicology 2005, 206, 207-219. [CrossRef] [PubMed]

3. Suzuki, T.; Iwahashi, Y. Comprehensive gene expression analysis of type B trichothecenes. J. Agric. Food Chem. 2012, 60, 9519-9527. [CrossRef] [PubMed]

4. McLaughlin, J.E.; Bin-Umer, M.A.; Tortora, A.; Mendez, N.; McCormick, S.; Tumer, N.E. A genome-wide screen in Saccharomyces cerevisiae reveals a critical role for the mitochondria in the toxicity of a trichothecene mycotoxin. Proc. Natl. Acad. Sci. USA 2009, 106, 21883-21888. [CrossRef] [PubMed]

5. Desjardins, A.E.; McCormick, S.P.; Appell, M. Structure-activity relationships of trichothecene toxins in an Arabidopsis thaliana leaf assay. J. Agric. Food Chem. 2007, 55, 6487-6492. [CrossRef] [PubMed]

6. Pestka, J.J. Deoxynivalenol: Mechanisms of action, human exposure, and toxicological relevance. Arch. Toxicol. 2010, 84, 663-679. [CrossRef] [PubMed]

7. Ward, T.J.; Clear, R.M.; Rooney, A.P.; O'Donnell, K.; Gaba, D.; Patrick, S.; Starkey, D.E.; Gilbert, J.; Geiser, D.M.; Nowicki, T.W. An adaptive evolutionary shift in Fusarium head blight pathogen populations is driving the 
rapid spread of more toxigenic Fusarium graminearum in North America. Fungal Genet. Biol. 2008, 45, 473-484. [CrossRef] [PubMed]

8. Alexander, N.J.; McCormick, S.P.; Ziegenhorn, S.L. Phytotoxicity of selected trichothecenes using Chlamydomonas reinhardtii as a model systemt. Nat. Toxins 1999, 7, 265-269. [CrossRef]

9. Pestka, J.J. Deoxynivalenol: Toxicity, mechanisms and animal health risks. Anim. Feed Sci. Technol. 2007, 137, 283-298. [CrossRef]

10. Alassane-Kpembi, I.; Kolf-Clauw, M.; Gauthier, T.; Abrami, R.; Abiola, F.A.; Oswald, I.P.; Puel, O. New insights into mycotoxin mixtures: The toxicity of low doses of Type B trichothecenes on intestinal epithelial cells is synergistic. Toxicol. Appl. Pharmacol. 2013, 272, 191-198. [CrossRef] [PubMed]

11. Suzuki, T.; Iwahashi, Y. Phytotoxicity evaluation of type B trichothecenes using a Chlamydomonas reinhardtii model system. Toxins 2014, 6, 453-463. [CrossRef] [PubMed]

12. Sundstøl Eriksen, G.; Pettersson, H.; Lundh, T. Comparative cytotoxicity of deoxynivalenol, nivalenol, their acetylated derivatives and de-epoxy metabolites. Food Chem. Toxicol. 2004, 42, 619-624. [CrossRef] [PubMed]

13. Gratz, S.W.; Duncan, G.; Richardson, A.J. The human fecal microbiota metabolizes deoxynivalenol and deoxynivalenol-3-glucoside and may be responsible for urinary deepoxy-deoxynivalenol. Appl. Environ. Microbiol. 2013, 79, 1821-1825. [CrossRef] [PubMed]

14. European Food Safety Authority (EFSA). Deoxynivalenol in Food And feed: Occurrence and Exposure. In EFSA Journal; EFSA: Parma, Italy, 2013; Volume 11, pp. 1-56.

15. Leonard, P.J.; Rathod, P.K.; Golin, J. Loss of function mutation in the yeast multiple drug resistance gene PDR5 causes a reduction in chloramphenicol efflux. Antimicrob. Agents Chemother. 1994, 38, 2492-2494. [CrossRef] [PubMed]

16. Mitterbauer, R.; Adam, G. Saccharomyces cerevisae and Arabidopsis thaliana: Useful model systems for the identification of molecular mechanisms involved in resistance of plants to toxins. Eur. J. Plant Pathol. 2002, 108, 699-703. [CrossRef]

17. Wehrschütz-Sigl, E.; Jungwirth, H.; Bergler, H.; Högenauer, G. The transporters Pdr5p and Snq2p mediate diazaborine resistance and are under the control of the gain-of-function allele PDR1-12. Eur. J. Biochem. 2004, 271, 1145-1152. [CrossRef] [PubMed]

18. Suzuki, T.; Sirisattha, S.; Mori, K.; Iwahashi, Y. Mycotoxin toxicity in Saccharomyces cerevisiae differs depending on gene mutations. Food Sci. Technol. Res. 2009, 6, 453-458. [CrossRef]

19. Suzuki, T.; Iwahashi, Y. Low toxicity of deoxynivalenol-3-glucoside in microbial cells. Toxins 2015, 7, 187-200. [CrossRef] [PubMed]

20. Mitterbauer, R.; Weindorfer, H.; Safaie, N.; Krska, R.; Lemmens, M.; Ruckenbauer, P.; Kuchler, K.; Adam, G. A sensitive and inexpensive yeast bioassay for the mycotoxin zearalenone and other compounds with estrogenic activity. Appl. Environ. Microbiol. 2003, 69, 805-811. [CrossRef] [PubMed]

21. Jungwirth, H.; Kuchler, K. Yeast ABC transporters-A tale of sex, stress, drugs and aging. FEBS Lett. 2006, 580, 1131-1138. [CrossRef] [PubMed]

22. Georgatsou, E.; Alexandraki, D. Regulated expression of the Saccharomyces cerevisiae Fre1p/Fre2p Fe/Cu reductase related genes. Yeast 1999, 15, 573-584. [CrossRef]

23. Georgatsou, E.; Alexandraki, D. Two distinctly regulated genes are required for ferric reduction, the first step of iron uptake in Saccharomyces cerevisiae. Mol. Cell. Biol. 1994, 14, 3065-3073. [CrossRef] [PubMed]

24. Shatwell, K.P.; Dancis, A.; Cross, A.R.; Klausner, R.D.; Segal, A.W. The FRE1 ferric reductase of Saccharomyces cerevisiae is a cytochrome $b$ similar to that of NADPH oxidase. J. Biol. Chem. 1996, 271, 14240-14244. [PubMed]

25. Hajji, K.; Clotet, J.; Ariño, J. Disruption and phenotypic analysis of seven ORFs from the left arm of chromosome XV of Saccharomyces cerevisiae. Yeast 1999, 15, 435-441. [CrossRef]

26. Suzuki, T.; Iwahashi, Y. Gene expression profile of MAP kinase PTC1 mutant exposed to Aflatoxin B1: Dysfunctions of gene expression in glucose utilization and sphingolipid metabolism. Chem. Biol. Informat. J. 2009, 9, 94-107. [CrossRef]

27. Soriano, J.M.; Gonzalez, L.; Catala, A.I. Mechanism of action of sphingolipids and their metabolites in the toxicity of fumonisin B1. Prog. Lipid Res. 2005, 44, 345-356. [CrossRef] [PubMed]

28. Cherkasova, V.; Lyons, D.M.; Elion, E.A. Fus3p and Kss1p control G1 arrest in Saccharomyces cerevisiae through a balance of distinct arrest and proliferative functions that operate in parallel with Far1p. Genetics 1999, 151, 989-1004. [PubMed] 
29. Ozcan, S.; Johnston, M. Three different regulatory mechanisms enable yeast hexose transporter (HXT) genes to be induced by different levels of glucose. Mol. Cell. Biol. 1995, 15, 1564-1572. [CrossRef] [PubMed]

30. Ozcan, S.; Johnston, M. Function and regulation of yeast hexose transporters. Microbiol. Mol. Biol. Rev. 1999, 63, 554-569. [PubMed]

31. Kim, J.H.; Roy, A.; Jouandot, D.; Cho, K.H. The glucose signaling network in yeast. Biochim. Biophys. Acta 2013, 1830, 5204-5210. [CrossRef] [PubMed]

32. Evaluations of the Joint FAO/WHO Expert Committee on Food Additives (JECFA). Available online: http:/ / apps.who.int/food-additives-contaminants-jecfa-database/chemical.aspx?chemID=2947 (accessed on 25 November 2015).

33. Saccharomyces Genome Database. Available online: http://www.yeastgenome.org/ (accessed on 15 September 2015).

34. Iwahashi, H.; Odani, M.; Ishidou, E.; Kitagawa, E. Adaptation of Saccharomyces cerevisiae to high hydrostatic pressure causing growth inhibition. FEBS Lett. 2005, 579, 2847-2852. [CrossRef] [PubMed]

35. Primer3. Available online: http:// frodo.wi.mit.edu/ (accessed on 6 April 2015).

36. Gene Expression Omnibus Database. Available online: http://www.ncbi.nlm.nih.gov/geo/ (accessed on 12 October 2015).

(C) 2016 by the authors; licensee MDPI, Basel, Switzerland. This article is an open access article distributed under the terms and conditions of the Creative Commons by Attribution (CC-BY) license (http:/ / creativecommons.org/licenses/by/4.0/). 